\title{
Diversidade e riqueza de formigas (Hymenoptera: Formicidae) em remanescentes de Mata Atlântica na Bacia Hidrográfica do Alto Tietê, SP
}

\author{
Silvia Sayuri Suguituru', Débora Rodrigues de Souza ${ }^{1}$, Catarina de Bortoli Munhae ${ }^{2}$, \\ Renata Pacheco $^{3}$ \& Maria Santina de Castro Morini ${ }^{1,4}$ \\ ${ }^{l}$ Núcleo de Ciências Ambientais -NCA, Laboratório de Mirmecologia, \\ Universidade de Mogi das Cruzes - UMC, Av. Dr. Cândido Xavier de Almeida e Souza, 200, \\ CEP 08701-970, Mogi das Cruzes, SP, Brasil. www.umc.br \\ ${ }^{2}$ Centro de Estudos de Insetos Sociais, Universidade Estadual Paulista - UNESP, \\ CEP 13506-900, Rio Claro, SP, Brasil. www.unesp.br \\ ${ }^{3}$ Instituto de Biologia, Universidade Federal de Uberlândia - UFU, \\ CEP 38400-90, Uberlândia, MG, Brasil. www.ufu.br \\ ${ }^{4}$ Autor para correspondência: Maria Santina de Castro Morini, e-mail: morini@umc.br
}

SUGUITURU, S.S., SOUZA, D.R., MUNHAE, C.B., PACHECO, R. \& MORINI, M.S.C. Ant species richness and diversity (Hymenoptera: Formicidae) in Atlantic Forest remnants in the Upper Tietê River Basin. Biota Neotrop. 13 (2): http://www.biotaneotropica.org.br/v13n2/en/abstract?inventory+bn00813022013

\begin{abstract}
The objective of this study was to describe the diversity, composition and similarity of the ant fauna in seven remnants of Atlantic Forest of Upper Tietê River Basin. Two of these remnants belong to Conservation Units, four are under the protection and a fragment belongs to a private property. In each area, $50 \mathrm{~m}^{2}$ litter samples were collected, which underwent mini-Winkler extractors, where they remained for $48 \mathrm{~h}$. All collections occurred during the rainy season. In total were recorded 11 subfamilies, 44 genera and 158 morphospecies/species of ants. The most frequent species in all areas were Pheidole sp.7, Solenopsis sp.1, Hypoponera sp.1 and Strumigenys denticulata. The highest $\alpha$ diversity value was recorded in the conservation unit with characteristic Atlantic Forest floristic composition, the lowest value in forests with anthropogenic influence. The variation in species composition between areas indicates the replacement of species among remnants of dense rain forest of the Alto Tietê River Basin, which suggests the importance of preserving these areas for the conservation of the regional ant fauna.

Keywords: communities of ants, Serra do Itapeti, litter, Rain Forest, inventory.
\end{abstract}

SUGUITURU, S.S., SOUZA, D.R., MUNHAE, C.B., PACHECO, R. \& MORINI, M.S.C. Diversidade e riqueza de formigas (Hymenoptera: Formicidae) em remanescentes de Mata Atlântica na Bacia Hidrográfica do Alto Tietê, SP. Biota Neotrop. 13 (2): http://www.biotaneotropica.org.br/v13n2/pt/abstract?inventory+bn00813022013

Resumo: O objetivo deste estudo foi descrever a diversidade, composição e a similaridade da fauna de formigas entre sete remanescentes de Floresta Ombrófila Densa da Bacia Hidrográfica do Alto Tietê. Dois desses remanescentes pertencem à Unidades de Conservação, quatro estão sob proteção e um fragmento pertence à propriedade particular. Em cada área foram coletadas $50 \mathrm{~m}^{2}$ de serapilheira, que foram submetidas à extratores do tipo mini-Winkler, onde permaneceram por $48 \mathrm{~h}$. Todas as coletas ocorreram no período chuvoso. No total foram registradas 11 subfamílias, 44 gêneros e 158 morfoespécies/espécies de formigas. As espécies mais frequentes em todas as áreas foram Pheidole sp.7, Solenopsis sp.1, Hypoponera sp.1 e Strumigenys denticulata. O maior valor de diversidade $\alpha$ foi registrado na unidade de conservação com composição florística de Mata Atlântica; o menor valor em florestas com influência antrópica. A variação na composição de espécies entre as áreas indica a substituição de espécies entre os remanescentes de Floresta Ombrófila Densa da Bacia Hidrográfica do Alto Tietê, o que sugere a importância da preservação dessas áreas para a conservação da diversidade regional da fauna de formigas.

Palavras-chave: comunidades de formigas, Serra do Itapeti, serapilheira, Floresta Ombrófila Densa, inventário. 


\section{Introdução}

O Estado de São Paulo possui atualmente cerca de 3,5 milhões de ha de cobertura vegetal natural, o que corresponde a $13,94 \%$ de sua superfície (Xavier et al. 2008). A Mata Atlântica brasileira, que já cobriu cerca de um milhão e duzentos mil quilômetros quadrados, está reduzida a $12 \%$ de sua área original (Ribeiro et al. 2009) e apenas 20\% está protegida (Câmara 2003). Considerada um dos hot spots em biodiversidade mais ameaçados do planeta (Fundação... 1998, Myers et al. 2000), a Mata Atlântica apresenta fauna e flora com altos níveis de endemismo (Martini et al. 2007, Carnaval et al. 2009, Metzger et al. 2009), formando um complexo de ecossistemas pertencentes ao Domínio Atlântico, dentre eles a fitofisionomia Floresta Ombrófila Densa (Joly et al. 1999). No Estado de São Paulo, a Floresta Ombrófila pode ser dividida em três formações: as matas de planície litorânea, as matas de encosta e as matas de altitude (Joly et al. 1992). Na Bacia Hidrográfica do Alto Tietê do Rio Tietê, a Floresta Ombrófila Densa ainda predomina na Serra do Mar e nas encostas da Serra do Itapeti, apesar de toda a pressão antrópica presente na região (Cetesb 1995, 1999, Marceniuk \& Hilsdorf 2010).

O Alto Tietê desempenha papel estratégico do ponto de vista ambiental, pois $64 \%$ de seu território estão inseridos em área de mananciais e seus municípios fazem parte da Reserva da Biosfera do Cinturão Verde da cidade de São Paulo (Pagani 2012). Dentre as áreas de conservação presente na região, encontram-se a Área de Proteção Ambiental da Várzea do Rio Tietê, a Área de Proteção da Serra do Itapeti, o Parque Estadual Nascentes do Rio Tietê, a Estação Biológica de Boracéia, a Estação Ecológica de Itapeti e o Parque Natural Municipal Francisco Affonso de Mello. Além da concentração de áreas para conservação ambiental, existem locais destinados ao lazer, turismo e produção agrícola. Tais atividades fazem do Alto Tietê uma importante região de serviços ecossistêmicos, econômicos e sociais que são fundamentais para a sustentabilidade da metrópole de São Paulo, como é o caso do abastecimento de água para milhões de pessoas (Candido et al. 2010).

Diversas funções são desempenhadas pelas formigas nos ecossistemas, como a ciclagem de nutrientes (Hölldobler \& Wilson 1990, Folgarait 1998), manutenção da morfologia do solo (Mckey et al. 2010) e interação com outros organismos (Del-Claro \& Oliveira 1999). Ao atuarem como engenheiras (Folgarait 1998, Sanders \& Van Veen 2011), as formigas alteram as propriedades estruturais e químicas do solo por meio da construção de galerias, aumentando a porosidade, drenagem, aeração, volume e incorporação da matéria orgânica (Hole 1981, Lavelle et al. 1997, Risch \& Jurgensen 2008, Sanders \& Van Veen 2011). Essas mudanças influenciam positivamente a taxa de decomposição e produtividade do sistema (Sanders \& Van Veen 2011).

As formigas possuem características importantes em estudos de biodiversidade por apresentarem alta diversidade, dominância numérica e de biomassa em quase todos os hábitats, facilidade na amostragem e identificação em morfoespécies e presença de ninhos estacionários, que permitem a re-amostragem ao longo do tempo (Alonso \& Agosti 2000). Além disso, muitos estudos mostram o papel de Formicidae como bioindicadores, por serem sensíveis às mudanças que ocorrem no ambiente (Majer 1983, Andersen \& Majer 2004, Stephens \& Wagner 2006, Majer et al. 2007, Pais \& Varanda 2010). Nas florestas tropicais até $50 \%$ da fauna de formigas podem estar associados à serapilheira (Delabie \& Fowler 1995), onde apresentam altos níveis de diversidade taxonômica, morfológica e funcional (Kaspari 1996, Agosti et al. 2000, Ward 2000, Silva \& Brandão 2010). Nesse estrato, as formigas encontram recursos para forrageamento e nidificação e um microclima favorável a seus requisitos ambientais (Andersen 1983, Carvalho \& Vasconcelos 1999,
Kaspari \& Weiser 2000, Campos et al. 2003, Armbrecht et al. 2004, Philpott \& Foster 2005).

Considerando que as estratégias para a conservação e conhecimento da biodiversidade são baseadas principalmente em composição florística e riqueza de espécies (Nogueira et al. 2008), que os levantamentos biológicos são fundamentais para promover a conservação de remanescentes florestais e as formigas como importantes componentes da biodiversidade tropical, este trabalho objetivou descrever a diversidade, composição e a similaridade da fauna de formigas entre diferentes remanescentes de Floresta Ombrófila Densa na Bacia Hidrográfica do Alto Tietê. Além disso, pretendemos apresentar uma lista das espécies de formigas de serapilheira da região.

\section{Material e Métodos}

\section{1. Áreas de estudo}

O trabalho foi realizado em sete remanescentes de Floresta Ombrófila Densa, localizados na Bacia Hidrográfica do Alto Tietê; especificamente nos municípios de Salesópolis, Biritiba Mirim e Mogi das Cruzes (Figura 1, Tabela 1). Os remanescentes das Barragens de Paraitinga, Biritiba Mirim, Ponte Nova e Ribeirão do Campo se localizam nas adjacências das áreas inundadas e pertencem à Serra do Mar. A fiscalização das três primeiras Barragens é realizada pelo Departamento Autônomo de Água e Esgoto (DAAE); da quarta Barragem pela Companhia de Saneamento Básico do Estado de São Paulo (SABESP). A entrada em todas as Barragens é permitida apenas para pesquisa, sendo controlada pelos referidos órgãos. O Parque Natural Municipal Francisco Affonso de Mello, localizado na Serra do Itapeti, é uma Unidade de Conservação (Lei 6.220 de 29/12/2008) sob a égide da Prefeitura Municipal de Mogi das Cruzes. O Parque Estadual Nascentes do Rio Tietê, localizado na Serra do Mar, é uma Unidade de Conservação (Decretos Estaduais 29.181/88 e 37.701/93) sob a administração do Departamento de Águas e Energia Elétrica (DAEE). A entrada em ambos os Parques é permitida para projetos de educação ambiental, pesquisadores e visitantes. A Fazenda Santo Alberto corresponde a uma propriedade particular onde ocorre criação de gado e plantio de eucalipto; está localizada na encosta norte da Serra do Itapeti e o fragmento de Mata Atlântica da propriedade não é fiscalizado.

\section{Coleta de dados}

As coletas foram realizadas no período chuvoso da região (Minuzzi et al. 2007), e em cada área foi demarcado um transecto de $1.200 \mathrm{~m}$ de comprimento, a partir de $200 \mathrm{~m}$ da borda do fragmento. Foram realizados 25 pontos amostrais ao longo desse transecto, distantes $50 \mathrm{~m}$ entre si. Em cada ponto coletou-se duas amostras de serapilheira, uma à esquerda e outra à direita do transecto, distantes $20 \mathrm{~m}$ uma da outra. Em cada ponto foi demarcada uma parcela de $1 \mathrm{~m}^{2}$ onde a serapilheira foi raspada, peneirada e colocada em sacos de tecido devidamente identificados. O material peneirado foi introduzido em mini-Winkler por 48 h (Agosti \& Alonso 2000, Bestelmeyer et al. 2000) para a extração das formigas.

O material foi inicialmente separado em subfamílias de acordo com a proposta de Bolton (2003), identificado em nível de gêneros e nomeado de acordo com Bolton et al. (2006); exceto para o grupo de gêneros de Prenolepis que segue a classificação de LaPolla et al. (2010). Em seguida, o material foi separado em morfoespécies comparando os espécimes com os da coleção de Formicidae do Alto Tietê (Universidade de Mogi das Cruzes). A sequência de numeração dos táxons segue a referida coleção. As espécies foram identificadas por comparação com exemplares depositados no Museu de Zoologia 


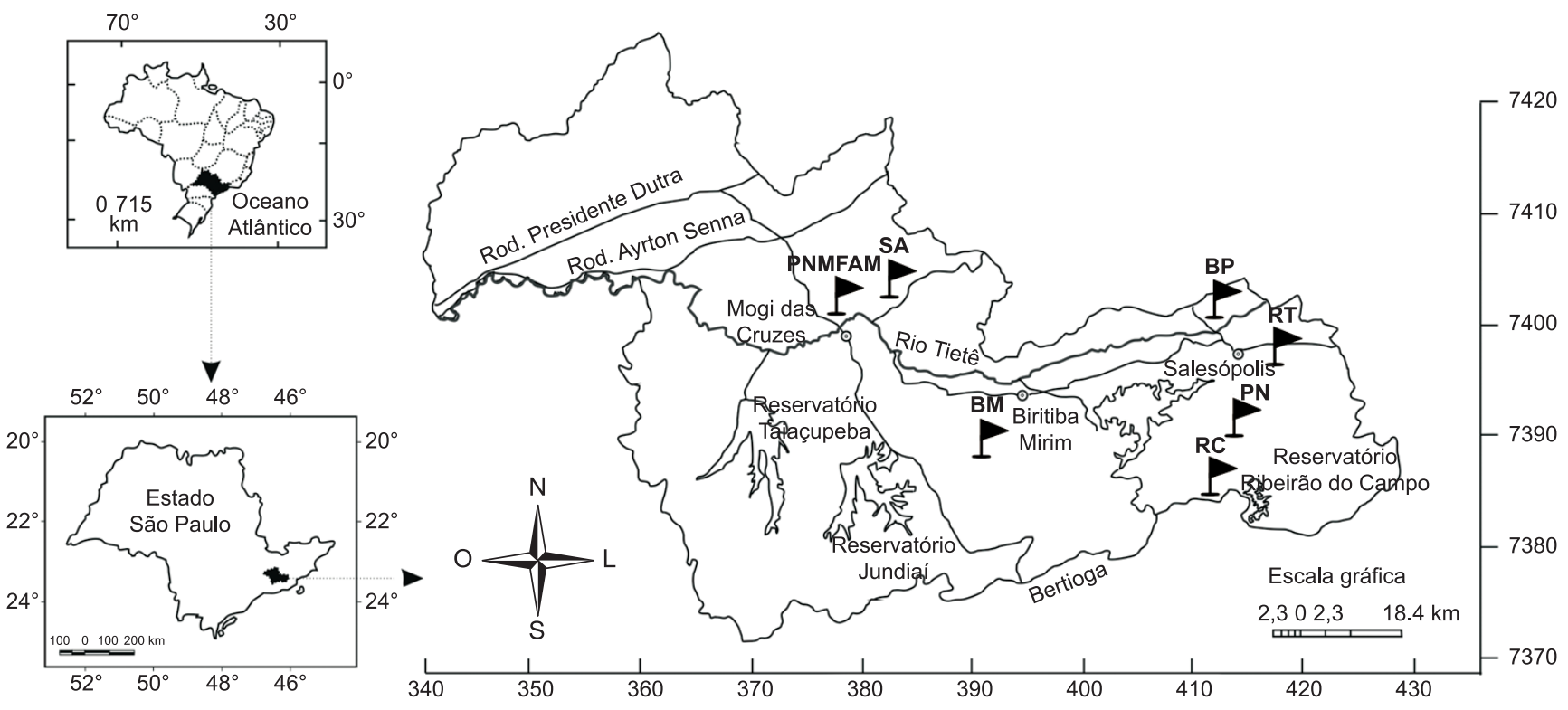

Figura 1. Localização geográfica da Bacia Hidrográfica do Alto Tietê, sendo destacados os remanescentes de Mata Atlântica onde o trabalho foi realizado (BP) Barragem de Paraitinga; (BM) Barragem de Biritiba Mirim; (PN) Barragem de Ponte Nova; (PNMFAM) Parque Natural Municipal Francisco Affonso de Mello; (RT) Parque Estadual Nascentes do Rio Tietê; (RC) Barragem de Ribeirão do Campo e (SA) Fazenda Santo Alberto.

Figure 1. Geographical location of Upper Tietê River Basin, highlighting the Atlantic Forest remnants where the study was performed. (BP) Paraitinga Dam; (BM) Biritiba Mirim Dam; (PN) Ponte Nova Dam; (PNMFAM) Municipal Natural Park Francisco Affonso de Mello; (RT) State Park Nascentes do Rio Tietê; (RC) Ribeirão do Campo Dam and (SA) Santo Alberto Farm.

Tabela 1. Locais de coleta com os respectivos códigos, localização geográfica e altitude.

Table 1. Sampling sites with respective codes, geographical location and altitude.

\begin{tabular}{|c|c|c|c|c|c|c|}
\hline \multirow{2}{*}{$\begin{array}{l}\text { Município } \\
\text { Salesópolis }\end{array}$} & \multirow{2}{*}{$\begin{array}{c}\text { Local } \\
\text { Barragem de Paraitinga }\end{array}$} & \multirow{2}{*}{$\begin{array}{c}\begin{array}{c}\text { Código de } \\
\text { coleta }\end{array} \\
\mathrm{BP}\end{array}$} & \multicolumn{2}{|c|}{ Localização geográfica } & \multirow{2}{*}{$\begin{array}{c}\begin{array}{c}\text { Altitude } \\
\text { (m) }\end{array} \\
823\end{array}$} & \multirow{2}{*}{$\begin{array}{c}\text { Tamanho } \\
\text { (ha)* }\end{array}$} \\
\hline & & & Serra do Mar & S 2331'9”; O 4656’8”' & & \\
\hline & Barragem de Ponte Nova & PN & & $\mathrm{S} 23^{\circ} 36^{\prime} 4^{\prime \prime} ; \mathrm{O} 45^{\circ} 58^{\prime} 10^{\prime \prime}$ & 812 & 200 \\
\hline & $\begin{array}{l}\text { Parque Estadual Nascentes do Rio } \\
\text { Tietê/UC** }\end{array}$ & RT & & S 2334'17’; O 4544'59”' & 804 & 134 \\
\hline & Barragem de Ribeirão do Campo & $\mathrm{RC}$ & & S 2334’10”; O 4549’57” & 753 & 200 \\
\hline $\begin{array}{l}\text { Biritiba } \\
\text { Mirim }\end{array}$ & Barragem de Biritiba Mirim & $\mathrm{BM}$ & & S 2329’22”; O 46'11'55" & 800 & 200 \\
\hline \multirow[t]{2}{*}{$\begin{array}{l}\text { Mogi das } \\
\text { Cruzes }\end{array}$} & $\begin{array}{l}\text { Parque Natural Municipal } \\
\text { Francisco Affonso de Mello/UC** }\end{array}$ & PNMFAM & Serra do Itapeti & S 2329’22”; O 46¹1'55" & 810 & 350 \\
\hline & Fazenda Santo Alberto & SA & & $\mathrm{S} 23^{\circ} 25^{\prime} 05^{\prime \prime} ; \mathrm{O} 46^{\circ} 10^{\prime} 48^{\prime \prime}$ & 690 & 130 \\
\hline
\end{tabular}

*tamanho aproximado da área do remanescente; **Unidade de Conservação.

da Universidade de São Paulo (MZSP), e com literatura pertinente. Os vouchers foram depositados na Universidade de Mogi das Cruzes (SP) e MZSP.

\section{Análise de dados}

A riqueza de formigas entre os remanescentes foi comparada por meio de curvas de rarefação (Gotelli 2009), baseadas no número de ocorrência das espécies. O número esperado de espécies, em cada remanescente, foi determinado usando o estimador de riqueza Chao 2; em ambas as análises foi usado o programa EstimateS, versão 8.2 (Colwell 2009). O teste de Kruskal-Wallis (Ayres et al. 2007) foi usado para comparar o número de espécies por $\mathrm{m}^{2}$ de serapilheira entre os diferentes remanescentes. A diversidade $\beta$ foi avaliada pelo índice de Jaccard, usando o pacote Vegan (Oksanen et al. 2011), que explora as relações de similaridade na composição de espécies de formigas entre os remanescentes e é influenciado pelo número de espécies de cada área (diversidade $\alpha$ ) e da região (diversidade $\gamma)$; pela razão entre diversidade local $(\alpha)$ e diversidade regional $(\gamma)$ (Chase et al. 2011) e, também, pelo índice de Whittaker (Whittaker 1960), que avalia a troca espacial de composição de espécies entre as comunidades locais. Este índice varia de 0 , quando duas amostras não apresentam nenhuma diferença na composição de espécies e 2, quando esta diferença é máxima, sendo calculado pela fórmula: $\beta=(\mathrm{c} / \mathrm{a})-1$, onde: $\mathrm{c}=$ total de espécies nos remanescentes amostrados; $\mathrm{a}=$ média do número de espécies dos remanescentes amostrados.

\section{Resultados}

Foram registradas em $350 \mathrm{~m}^{2}$ de serapilheira, 11 subfamílias, 43 gêneros e 158 morfoespécies/espécies (diversidade $\gamma$ ou regional) de formigas (173 espécies estimadas); tendo sido encontrada uma nova espécie (Strumigenys sp.9) (Tabela 2). Myrmicinae foi a subfamília 
Tabela 2. Número de ocorrência, Frequência relativa (\%), riqueza e estimativa de riqueza das espécies de formigas de serapilheira capturadas com extrator de mini-Winkler em remanescentes de Mata Atlântica localizados no Alto Tietê (SP). (BP) Barragem de Paraitinga; (BM) Barragem de Biritiba Mirim; (PN) Barragem de Ponte Nova; (PNMFAM) Parque Natural Municipal Francisco Affonso de Mello; (RT) Parque Estadual Nascentes do Rio Tietê; (RC) Barragem de Ribeirão do Campo e (SA) Fazenda Santo Alberto.

Table 2. Number of occurrence and relative frequency (\%) of species of litter ants caught using mini-Winkler extractor in remnants of Atlantic Forest located in the Upper Tietê (São Paulo State). (BP) Paraitinga Dam; (BM) Biritiba Mirim Dam; (PN) Ponte Nova Dam; (PNMFAM) Municipal Natural Park Francisco Affonso de Mello; (RT) State Park Nascentes do Rio Tietê; (RC) Ribeirão do Campo Dam and (SA) Santo Alberto Farm.

\begin{tabular}{|c|c|c|c|c|c|c|c|}
\hline Subfamílias/espécies & $\mathbf{B P}$ & $\mathbf{B M}$ & PN & PNMFAM & RT & $\mathbf{R C}$ & SA \\
\hline \multicolumn{8}{|l|}{ Amblyoponinae } \\
\hline Prionopelta antillana Forel, 1909 & - & $3(0,39)$ & $1(0,14)$ & $2(0,36)$ & - & - & - \\
\hline Stigmatomma elongatum Santschi, 1912 & - & $2(0,26)$ & - & - & - & - & - \\
\hline Stigmatomma armigera Mayr, 1887 & - & $1(0,13)$ & - & - & - & $1(0,17)$ & - \\
\hline \multicolumn{8}{|l|}{ Cerapachyinae } \\
\hline Cerapachys sp.1 & - & - & - & - & $3(0,69)$ & - & - \\
\hline \multicolumn{8}{|l|}{ Dolichoderinae } \\
\hline Dorymyrmex sp.1 & - & - & - & $1(0,18)$ & - & - & - \\
\hline Linepithema iniquum (Mayr, 1870) & - & - & $2(0,28)$ & $1(0,18)$ & - & $4(0,66)$ & - \\
\hline Linepithema neotropicum Wild, 2007 & $6(0,81)$ & $3(0,39)$ & $2(0,28)$ & $5(0,9)$ & $1(0,23)$ & $5(0,83)$ & - \\
\hline \multicolumn{8}{|l|}{ Ecitoninae } \\
\hline Eciton burchelli Westwood, 1842 & - & $3(0,39)$ & - & - & - & - & - \\
\hline Labidus coecus (Latreille, 1802) & - & - & - & $2(0,36)$ & $2(0,46)$ & - & - \\
\hline Labidus praedator (Smith F., 1858) & - & - & - & $1(0,18)$ & $3(0,69)$ & - & - \\
\hline \multicolumn{8}{|l|}{ Ectatomminae } \\
\hline Gnamptogenys continua (Mayr, 1887) & $2(0,28)$ & $2(0,26)$ & $2(0,28)$ & $1(0,18)$ & - & - & $1(0,18)$ \\
\hline Gnamptogenys reichenspergeri (Santschi, 1929) & - & - & - & $1(0,18)$ & - & $2(0,34)$ & - \\
\hline Gnamptogenys striatula Mayr, 1884 & $18(2,45)$ & $21(2,73)$ & $33(4,89)$ & $16(2,90)$ & $15(3,43)$ & $25(4,17)$ & $15(2,75)$ \\
\hline Gnamptogenys minuta (Emery, 1896) & - & $1(0,13)$ & - & - & - & - & - \\
\hline Gnamptogenys sp.6 & - & - & - & - & - & - & $4(0,74)$ \\
\hline Typhlomyrmex rogenhoferi Mayr, 1862 & - & $1(0,13)$ & - & - & $4(0,9)$ & - & - \\
\hline Typholomyrmex major Santschi, 1923 & $1(0,14)$ & - & - & - & - & - & - \\
\hline \multicolumn{8}{|l|}{ Formicinae } \\
\hline Camponotus crassus Mayr, 1862 & $1(0,14)$ & - & - & $1(0,18)$ & - & - & - \\
\hline Camponotus rufipes (Fabricius, 1775) & - & $1(0,13)$ & - & $3(0,54)$ & $2(0,46)$ & - & - \\
\hline $\begin{array}{l}\text { Camponotus sericeiventris (Guérin-Méneville, } \\
\text { 1838) }\end{array}$ & - & - & $1(0,14)$ & $2(0,36)$ & - & - & - \\
\hline Camponotus (Taenamyrmex) sp.1 & - & - & - & $1(0,18)$ & $2(0,46)$ & - & - \\
\hline Camponotus sp.2 & - & - & - & - & - & $1(0,17)$ & - \\
\hline Camponotus sp.6 & - & - & - & $1(0,18)$ & - & - & - \\
\hline Camponotus sp.8 & $1(0,14)$ & - & - & $1(0,18)$ & - & - & - \\
\hline Camponotus sp.9 & $1(0,14)$ & - & - & - & - & - & - \\
\hline Camponotus sp.10 & - & $1(0,13)$ & - & $3(0,54)$ & - & $3(0,5)$ & - \\
\hline Brachymyrmex heeri Forel, 1874 & $18(2,45)$ & $6(0,78)$ & $18(2,61)$ & $29(5,3)$ & $5(1,14)$ & $16(2,67)$ & $11(2,02)$ \\
\hline Brachymyrmex incisus Forel, 1912 & $1(0,14)$ & $5(0,65)$ & $5(0,72)$ & $2(0,36)$ & $16(3,67)$ & $10(1,67)$ & $14(2,57)$ \\
\hline Brachymyrmex luederwaldti Santschi, 1923 & - & - & $1(0,14)$ & - & $1(0,23)$ & - & - \\
\hline Brachymyrmex micromegas Emery, 1923 & - & $1(0,13)$ & - & $1(0,18)$ & - & - & $2(0,36)$ \\
\hline Brachymyrmex pictus Mayr, 1887 & $18(2,45)$ & $23(2,99)$ & $4(0,58)$ & $17(3,10)$ & $5(1,14)$ & $19(3,16)$ & $17(3,12)$ \\
\hline Myrmelachista arthuri Forel, 1903 & - & - & - & $2(0,36)$ & - & - & - \\
\hline Myrmelachista catharinae Mayr, 1887 & $1(0,14)$ & - & $1(0,14)$ & $1(0,18)$ & - & - & - \\
\hline Myrmelachista sp.4 & - & - & - & $1(0,18)$ & - & $1(0,17)$ & - \\
\hline Nylanderia fulva Mayr, 1862 & $11(1,50)$ & $18(2,34)$ & $27(3,92)$ & $12(2,17)$ & $21(4,9)$ & $17(2,84)$ & $1(0,18)$ \\
\hline \multicolumn{8}{|l|}{ Heteroponerinae } \\
\hline Heteroponera dentinodis (Mayr, 1887) & $4(0,55)$ & $1(0,13)$ & $2(0,28)$ & $1(0,18)$ & $3(0,69)$ & $5(0,83)$ & $3(0,55)$ \\
\hline Heteroponera dolo (Roger, 1860) & - & - & $1(0,14)$ & - & - & - & - \\
\hline Heteroponera mayri Kempf, 1962 & $1(0,14)$ & $10(1,3)$ & $5(0,72)$ & $6(1,08)$ & $10(2,31)$ & $20(3,31)$ & - \\
\hline \multicolumn{8}{|l|}{ Myrmicinae } \\
\hline Acanthognathus ocellatus (Mayr, 1887) & - & $2(0,26)$ & $4(0,60)$ & - & - & - & - \\
\hline Acanthognathus rudis Brown e Kempf, 1969 & $5(0,68)$ & $6(0,78)$ & - & $3(0,54)$ & $3(0,69)$ & $3(0,5)$ & $3(0,55)$ \\
\hline
\end{tabular}

*- espécie não descrita. 
Tabela 2. Continuação...

\begin{tabular}{|c|c|c|c|c|c|c|c|}
\hline $\begin{array}{c}\text { Subfamílias/espécies } \\
\end{array}$ & BP & BM & PN & PNMFAM & RT & RC & SA \\
\hline Acromyrmex crassispinus (Forel, 1909) & - & - & - & $2(0,36)$ & - & $1(0,17)$ & - \\
\hline Acromyrmex niger (Smith F., 1858) & $3(0,41)$ & $1(0,13)$ & $1(0,14)$ & $1(0,18)$ & $1(0,23)$ & $1(0,17)$ & - \\
\hline Apterostigma sp.1 & - & $2(0,26)$ & - & $1(0,18)$ & - & - & - \\
\hline Apterostigma sp. 2 & - & - & $11(1,60)$ & - & - & - & - \\
\hline Apterostigma sp.3 & - & - & - & - & $1(0,23)$ & $2(0,34)$ & $3(0,55)$ \\
\hline Atta sexdens (Linnaeus, 1758) & - & $2(0,26)$ & - & $1(0,18)$ & - & - & $3(0,55)$ \\
\hline Basiceros disciger (Mayr, 1887) & - & $3(0,39)$ & - & $2(0,36)$ & - & - & - \\
\hline Basiceros rugiferum (Mayr, 1887) & $15(2,05)$ & $21(2,75)$ & $11(1,60)$ & $18(3,25)$ & $4(0,92)$ & $9(1,5)$ & $1(0,18)$ \\
\hline Basiceros spectabile (Kempf, 1962) & - & - & $2(0,28)$ & - & - & - & - \\
\hline Basiceros stenognathum (Brown e Kempf, 1960) & $13(1,77)$ & $27(3,51)$ & $20(2,90)$ & $17(3,10)$ & $6(1,37)$ & $19(3,16)$ & $1(0,18)$ \\
\hline Basiceros sp.4 & $1(0,14)$ & - & - & - & - & - & - \\
\hline Carebara sp.1 & $1(0,14)$ & - & $10(1,45)$ & - & - & $12(2)$ & $1(0,18)$ \\
\hline $\begin{array}{l}\text { Crematogaster (Neocrema) magnifica Santschi, } \\
1925\end{array}$ & - & - & $2(0,28)$ & $1(0,18)$ & - & - & - \\
\hline Crematogaster sp.1 (Gr. Orthocrema) & $8(1,09)$ & $3(0,39)$ & $3(0,43)$ & $13(2,35)$ & $1(0,23)$ & - & - \\
\hline Crematogaster sp.2 (Gr. Orthocrema) & - & - & $1(0,14)$ & $1(0,18)$ & - & - & - \\
\hline Crematogaster sp. 3 & - & - & $3(0,43)$ & - & - & - & - \\
\hline Crematogaster sp.5 & - & - & $1(0,14)$ & - & - & - & - \\
\hline Crematogaster sp.7 & - & - & - & - & $4(0,92)$ & $5(0,83)$ & $1(0,18)$ \\
\hline Cyphomyrmex strigatus Mayr, 1887 & $1(0,14)$ & - & - & $1(0,18)$ & - & - & - \\
\hline Cyphomyrmex pr strigatus & $2(0,28)$ & - & - & $2(0,36)$ & - & - & $1(0,18)$ \\
\hline Hylomyrma balzani (Emery, 1894) & $13(1,77)$ & $5(0,65)$ & - & $3(0,54)$ & $11(2,54)$ & $8(1,34)$ & $18(3,3)$ \\
\hline Hylomyrma reitteri (Mayr, 1887) & $14(1,91)$ & $26(3,41)$ & $19(2,76)$ & $6(1,08)$ & $9(2,1)$ & $22(3,67)$ & $7(1,28)$ \\
\hline Lachnomyrmex plaumanni Borgmeir, 1957 & $4(0,55)$ & $2(0,26)$ & - & $2(0,36)$ & - & $4(0,67)$ & - \\
\hline Lachnomyrmex sp.1 & $1(0,14)$ & - & - & - & - & $7(1,17)$ & - \\
\hline Megalomyrmex goeldii Forel, 1912 & $16(2,18)$ & $11(1,43)$ & - & $2(0,36)$ & - & - & $5(0,92)$ \\
\hline Megalomyrmex iheringi Forel, 1911 & - & $1(0,13)$ & $20(2,90)$ & - & - & - & - \\
\hline Mycetarotes senticosus Kempf, 1960 & - & $1(0,13)$ & $2(0,28)$ & - & - & - & - \\
\hline Mycetosoritis sp.1 & $24(3,28)$ & $36(4,68)$ & $29(4,21)$ & $20(3,61)$ & $16(3,69)$ & $19(3,16)$ & $28(5,14)$ \\
\hline $\begin{array}{l}\text { Oxyepoecus myops Alburquerque \& Brandão, } \\
2009\end{array}$ & $3(0,41)$ & $12(1,57)$ & $11(1,60)$ & $8(1,45)$ & $2(0,46)$ & - & - \\
\hline Oxyepoecus rastratus (Mayr, 1887) & - & - & $1(0,14)$ & - & - & - & - \\
\hline Pheidole aberrans Mayr, 1868 & - & - & $1(0,14)$ & $1(0,18)$ & - & $1(0,17)$ & - \\
\hline Pheidole sp.4 & - & - & - & $1(0,18)$ & $1(0,23)$ & $2(0,34)$ & - \\
\hline Pheidole sp.5 & - & - & - & $1(0,18)$ & $30(6,92)$ & $28(4,67)$ & - \\
\hline Pheidole sp.6 & - & $11(1,43)$ & $1(0,14)$ & $6(1,08)$ & $2(0,46)$ & $13(2,17)$ & $21(3,85)$ \\
\hline Pheidole sp.7 & $50(6,83)$ & $36(4,68)$ & $41(6,0)$ & $34(6,15)$ & $1(0,23)$ & $8(1,34)$ & $19(3,49)$ \\
\hline Pheidole sp.8 & - & - & - & $1(0,18)$ & - & - & - \\
\hline Pheidole sp.9 & $2(0,28)$ & - & $2(0,28)$ & $1(0,18)$ & - & - & - \\
\hline Pheidole sp.10 & - & - & $4(0,58)$ & - & - & - & - \\
\hline Pheidole sp.11 & - & - & - & $3(0,54)$ & - & - & - \\
\hline Pheidole sp.12 & - & - & $4(0,58)$ & - & $13(3,00)$ & $8(1,34)$ & - \\
\hline Pheidole sp.13 & $27(3,68)$ & $36(4,68)$ & $5(0,72)$ & $20(3,61)$ & $1(0,23)$ & $5(0,83)$ & $16(2,93)$ \\
\hline Pheidole sp.14 & $2(0,28)$ & $10(1,3)$ & $1(0,14)$ & $10(1,8)$ & - & $8(1,34)$ & - \\
\hline Pheidole sp.15 & $2(0,28)$ & $2(0,26)$ & $9(1,31)$ & $5(0,9)$ & $7(1,62)$ & $2(0,34)$ & $27(4,95)$ \\
\hline Pheidole sp.16 & $27(3,68)$ & $7(0,91)$ & $4(0,58)$ & $7(1,26)$ & $5(1,14)$ & $5(0,83)$ & $12(2,2)$ \\
\hline Pheidole sp.17 & - & - & - & - & - & $2(0,34)$ & - \\
\hline Pheidole sp.18 & $1(0,14)$ & $4(0,52)$ & - & $1(0,18)$ & - & $1(0,17)$ & $1(0,18)$ \\
\hline Pheidole sp.19 & - & - & - & $1(0,18)$ & - & $1(0,17)$ & $1(0,18)$ \\
\hline Pheidole sp.20 & $3(0,41)$ & - & $3(0,43)$ & $5(0,9)$ & $2(0,46)$ & - & - \\
\hline Pheidole sp.21 & $1(0,14)$ & - & - & $2(0,36)$ & - & $2(0,34)$ & $1(0,18)$ \\
\hline Pheidole sp.22 & $15(2,05)$ & $10(1,3)$ & $3(0,43)$ & $7(1,26)$ & $1(0,23)$ & $3(0,5)$ & $8(1,47)$ \\
\hline Pheidole sp.23 & $3(0,41)$ & - & $7(1,01)$ & $2(0,36)$ & - & - & - \\
\hline Pheidole sp.24 & - & - & - & $1(0,18)$ & - & - & - \\
\hline
\end{tabular}

*- espécie não descrita. 
Tabela 2. Continuação...

\begin{tabular}{|c|c|c|c|c|c|c|c|}
\hline Subfamílias/espécies & BP & BM & PN & PNMFAM & RT & RC & SA \\
\hline Pheidole sp.26 & - & - & $2(0,28)$ & $2(0,36)$ & - & - & $1(0,18)$ \\
\hline Pheidole sp.28 & - & - & $8(1,16)$ & $4(0,72)$ & - & $6(1,0)$ & - \\
\hline Pheidole sp.29 & $1(0,14)$ & - & - & $1(0,18)$ & - & - & - \\
\hline Pheidole sp.30 & $4(0,55)$ & $2(0,26)$ & $1(0,14)$ & $2(0,36)$ & - & - & - \\
\hline Pheidole sp.31 & - & $7(0,91)$ & $6(0,87)$ & $1(0,18)$ & - & $2(0,34)$ & - \\
\hline Pheidole sp.32 & $2(0,28)$ & - & - & $1(0,18)$ & - & $7(1,17)$ & - \\
\hline Pheidole sp.33 & $1(0,14)$ & - & - & $2(0,36)$ & - & $1(0,17)$ & - \\
\hline Pheidole sp.34 & - & - & - & - & $3(0,69)$ & $2(0,34)$ & - \\
\hline Pheidole sp.35 & - & - & - & $1(0,18)$ & - & - & - \\
\hline Pheidole sp.36 & $1(0,14)$ & - & $1(0,14)$ & $1(0,18)$ & - & - & - \\
\hline Pheidole sp.38 & $4(0,55)$ & $1(0,13)$ & - & $1(0,18)$ & - & $1(0,17)$ & - \\
\hline Pheidole sp.39 & $3(0,41)$ & $1(0,13)$ & $3(0,43)$ & $1(0,18)$ & $3(0,69)$ & $8(1,34)$ & - \\
\hline Pogonomyrmex naegelii Gallardo, 1932 & - & - & - & - & - & $1(0,17)$ & - \\
\hline Pogonomyrmex sp.2 & - & - & - & - & - & $1(0,17)$ & - \\
\hline Procryptocerus pr. schmalzi & - & - & $1(0,14)$ & $1(0,18)$ & $1(0,23)$ & - & - \\
\hline Strumigenys appretiatus (Borgmeir, 1954) & - & - & - & $3(0,54)$ & - & $1(1,17)$ & $3(0,55)$ \\
\hline Strumigenys crassicornis Mayr, 1887 & $20(2,73)$ & $30(3,9)$ & $20(2,90)$ & $7(1,26)$ & $10(2,31)$ & $8(1,34)$ & $15(2,75)$ \\
\hline Strumigenys denticulata Mayr, 1887 & $47(6,42)$ & $46(5,98)$ & $47(6,82)$ & $4(0,72)$ & $30(6,92)$ & $16(2,67)$ & $36(6,6)$ \\
\hline Strumigenys eggersi (Emery, 1890) & $1(0,14)$ & - & $1(0,14)$ & - & - & - & $1(0,18)$ \\
\hline Strumigenys elongata Roger, 1863 & - & $3(0,39)$ & $11(1,60)$ & $8(1,45)$ & $3(0,69)$ & $1(0,17)$ & $6(1,1)$ \\
\hline Strumigenys louisianae Roger, 1863 & $7(0,95)$ & $2(0,26)$ & $1(0,14)$ & $11(1,99)$ & - & - & - \\
\hline Strumigenys schmalzi Emery, 1906 & $8(1,09)$ & $2(0,26)$ & $9(1,31)$ & - & - & $1(0,17)$ & - \\
\hline Strumigenys splendens (Borgmeir, 1954) & $1(0,14)$ & - & - & - & - & - & - \\
\hline Strumigenys sp.6 & $1(0,14)$ & - & $1(0,14)$ & $2(0,36)$ & - & $1(0,17)$ & - \\
\hline *Strumigenys sp. 9 & - & - & - & $2(0,36)$ & $2(0,46)$ & - & - \\
\hline Strumigenys sp.11 (Gr. Smithistruma) & $7(0,95)$ & $1(0,13)$ & - & $2(0,36)$ & - & - & - \\
\hline Strumigenys sp.12 & $1(0,14)$ & - & - & - & - & - & - \\
\hline Strumigenys sp.15 & - & - & - & - & - & $4(0,67)$ & - \\
\hline Rophalothrix sp.1 & $1(0,14)$ & - & - & - & - & - & - \\
\hline Solenopsis saevissima (Smith, 1855) & - & - & - & $5(0,9)$ & - & - & - \\
\hline Solenopsis wasmannii Emery, 1894 & $24(3,28)$ & $28(3,64)$ & $15(2,18)$ & $14(2,53)$ & $23(5,31)$ & $22(3,67)$ & $20(3,67)$ \\
\hline Solenopsis sp.1 & $50(6,83)$ & $49(6,43)$ & $45(6,53)$ & $48(8,68)$ & $33(7,6)$ & $33(5,4)$ & $49(9)$ \\
\hline Solenopsis sp.4 & $35(4,78)$ & $32(4,16)$ & $37(5,15)$ & $16(2,90)$ & $13(3,0)$ & $20(3,3)$ & $42(7,71)$ \\
\hline Solenopsis sp.5 & $1(0,14)$ & - & - & - & - & $4(0,67)$ & - \\
\hline Solenopsis sp.7 & - & - & - & - & - & $1(0,17)$ & - \\
\hline Solenopsis sp. 8 & $18(2,45)$ & - & - & - & - & - & - \\
\hline Trachymyrmex sp.1 (Gr. Septentrionales) & - & - & - & $1(0,18)$ & - & - & - \\
\hline Wasmannia sp.3 & $46(6,28)$ & $44(5,72)$ & $18(2,61)$ & $26(4,70)$ & $15(3,43)$ & $17(2,84)$ & $28(5,14)$ \\
\hline \multicolumn{8}{|l|}{ Ponerinae } \\
\hline Anochetus altisquamis Mayr, 1887 & $3(0,41)$ & $1(0,13)$ & $1(0,14)$ & $1(0,18)$ & $1(0,23)$ & $3(0,5)$ & - \\
\hline Anochetus neglectus Emery, 1894 & - & $2(0,26)$ & $1(0,14)$ & - & - & - & - \\
\hline Hypoponera sp.1 & $41(5,60)$ & $38(4,94)$ & $35(5,18)$ & $19(3,43)$ & $7(1,62)$ & $14(2,34)$ & $38(6,97)$ \\
\hline Hypoponera sp.2 & $2(0,28)$ & $13(1,69)$ & $13(1,88)$ & $2(0,36)$ & - & $2(0,34)$ & $6(1,1)$ \\
\hline Hypoponera sp.3 & - & $5(0,65)$ & $6(0,87)$ & $5(0,9)$ & $1(0,23)$ & $1(0,17)$ & $4(0,74)$ \\
\hline Hyроропеra sp.4 & $14(1,91)$ & $4(0,52)$ & $6(0,87)$ & $10(1,8)$ & $7(1,62)$ & $8(1,34)$ & $11(2,02)$ \\
\hline Hypoponera sp.5 & $3(0,41)$ & $2(0,26)$ & $3(0,43)$ & $1(0,18)$ & - & $1(0,17)$ & $1(0,18)$ \\
\hline Hypoponera sp.6 & $1(0,14)$ & $7(0,91)$ & $2(0,28)$ & $5(0,9)$ & $6(1,37)$ & $15(2,5)$ & $8(1,47)$ \\
\hline Hypoponera sp.7 & $1(0,14)$ & $7(0,91)$ & $5(0,72)$ & $4(0,72)$ & $7(1,62)$ & $14(2,34)$ & $4(0,74)$ \\
\hline Hyроропеra sp.8 & $1(0,14)$ & $37(4,81)$ & $17(2,47)$ & $2(0,36)$ & $31(7,12)$ & $35(5,71)$ & $5(0,92)$ \\
\hline Hуроропеra sp.9 & $16(2,18)$ & $11(1,43)$ & $6(0,87)$ & $4(0,72)$ & $6(1,37)$ & $3(0,5)$ & $7(1,28)$ \\
\hline Hуроропеra sp.10 & - & $1(0,13)$ & - & $1(0,18)$ & $1(0,23)$ & $1(0,17)$ & - \\
\hline Hypoponera sp.11 & $1(0,14)$ & - & $2(0,28)$ & - & - & $1(0,17)$ & - \\
\hline Hуроропеra sp.12 & - & - & - & - & $7(1,62)$ & $4(0,67)$ & $1(0,18)$ \\
\hline Hyроропеra sp.13 & $2(0,28)$ & - & - & - & $1(0,23)$ & $5(0,83)$ & - \\
\hline
\end{tabular}

\footnotetext{
*- espécie não descrita.
} 
Tabela 2. Continuação...

\begin{tabular}{|c|c|c|c|c|c|c|c|}
\hline Subfamílias/espécies & BP & BM & PN & PNMFAM & RT & $\mathbf{R C}$ & SA \\
\hline Hypoponera sp.14 & - & - & - & - & - & $1(0,17)$ & - \\
\hline Hypoponera sp.15 & - & $2(0,26)$ & - & - & - & - & - \\
\hline Hypoponera sp.16 & $1(0,14)$ & - & - & - & - & - & - \\
\hline Odontomachus affinis Guérin-Méneville, 1844 & $1(0,14)$ & - & $3(0,43)$ & - & - & - & $1(0,18)$ \\
\hline Odontomachus meinerti Forel, 1905 & $14(1,91)$ & $5(0,65)$ & $1(0,14)$ & - & - & - & $8(1,47)$ \\
\hline Pachycondyla constricta (Mayr, 1884) & $1(0,14)$ & - & - & - & $3(0,69)$ & $1(0,17)$ & - \\
\hline Pachycondyla harpax (Fabricius, 1804) & - & $1(0,13)$ & - & - & $8(1,85)$ & - & - \\
\hline Pachycondyla laevigata (Smith F., 1858) & - & - & - & $1(0,18)$ & - & - & - \\
\hline Pachycondyla striata Smith, 1858 & $1(0,14)$ & $1(0,13)$ & $3(0,43)$ & $1(0,18)$ & - & $1(0,17)$ & - \\
\hline \multicolumn{8}{|l|}{ Proceratiinae } \\
\hline Discothyrea neotropica Bruch, 1919 & - & - & $2(0,28)$ & - & - & $1(0,17)$ & - \\
\hline Discothyrea sexarticulata Borgmeier, 1954 & $4(0,55)$ & $4(0,52)$ & $14(2,03)$ & $1(0,18)$ & - & $1(0,17)$ & $4(0,74)$ \\
\hline \multicolumn{8}{|l|}{ Pseudomyrmecinae } \\
\hline Pseudomyrmex gracilis (Fabricius, 1804) & - & - & - & $1(0,18)$ & $1(0,18)$ & - & - \\
\hline Pseudomyrmex oculatus (Smith, 1855) & - & - & - & $1(0,18)$ & $1(0,18)$ & - & - \\
\hline Riqueza/remanescente (diversidade $\alpha$ ) & 81 & 73 & 81 & 105 & 62 & 83 & 53 \\
\hline Riqueza total (diversidade $\gamma$ ) & & & & 158 & & & \\
\hline Estimativa de riqueza/remanescente & 123,63 & 82,52 & 93,3 & 149,25 & 76,7 & 114,85 & 104,45 \\
\hline Estimativa de riqueza total & & & & 173 & & & \\
\hline
\end{tabular}

*- espécie não descrita.

mais rica em todos os remanescentes, com 93 espécies no total. Foram registradas 24 espécies para Ponerinae e 18 para Formicinae. Os gêneros mais ricos foram Pheidole e Hypoponera, com 34 e 16 espécies, respectivamente. Solenopsis sp.1 e Strumigenys denticulata exibem forte dominância na serapilheira dos remanescentes de Floresta Ombrófila Densa do Alto Tietê (Figura 2).

A diversidade $\alpha$ variou de 53 a 105 espécies. O Parque Natural Municipal Francisco Affonso de Mello possui a maior riqueza com 105 espécies (70,4\% do esperado) (Figura 3), seguido da Barragem de Ribeirão do Campo, com 83 espécies (71,4\% do esperado) (Tabela 2). Os remanescentes com menor riqueza pertencem ao Parque Estadual Nascentes do Rio Tietê, com 62 espécies $(80,8 \%$ do esperado) de formigas, e à Fazenda Santo Alberto, com 53 espécies (50,7\% do esperado). Nos demais remanescentes foram registradas 81 espécies (Barragem de Paraitinga; 65,4\% do esperado), na Barragem de Ponte Nova (81 espécies; $85,7 \%$ do esperado) e na Barragem de Biritiba Mirim ( 73 espécies; $88,5 \%$ do esperado). Houve diferença significativa no número de espécies por $\mathrm{m}^{2}$ de serapilheira entre os remanescentes (Kruskal-Wallis $=95,6034, d f=6, p<0,05$ ); o número médio de espécies por $\mathrm{m}^{2}$ de serapilheira/área variou de 9,23 $(\mathrm{dp} \pm 3,23)$ a $15,32(\mathrm{dp} \pm 4,70)$, sendo menor no Parque Estadual Nascentes do Rio Tietê e maior na Barragem de Biritiba Mirim (Figura 4).

A troca espacial de composição de espécies entre as comunidades locais é baixa, variando de 0,28 a 0,43 (Tabela 3 ). Remanescentes que estão sob proteção ambiental abrigam de 46 a $66 \%$ do total de espécies da região; a razão entre diversidade $\alpha$ e $\gamma$ varia entre 33 a $39 \%$ em florestas com interferência antrópica atual (Fazenda Santo Alberto) ou no passado (Parque Estadual Nascentes do Rio Tietê), respectivamente. Ocorre compartilhamento acentuado de espécies entre a maior Unidade de Conservação da Bacia Hidrográfica do Alto Tietê e as áreas de barragens (PNMFAM X BP: 62 espécies; PNMFAM X PN: 61 espécies; PNMFAM X RC: 60 espécies) (Tabela 3). As áreas de Floresta Ombrófila Densa da Barragem de Ribeirão do Campo e do Parque Estadual Nascentes do Rio Tietê são similares em relação à composição de espécies. Para as demais áreas, a maior similaridade está entre as formigas do Parque Natural Municipal Francisco Affonso de Mello e as Barragens de Ponte Nova, Paraitinga e Biritiba Mirim. As comunidades de formigas de serapilheira do fragmento de Floresta Ombrófila Densa pertencente à Fazenda Santo Alberto são as mais dissimilares (Figura 5).

\section{Discussão}

Os resultados aqui apresentados indicam que a riqueza de formigas de serapilheira é semelhante a outros remanescentes de Floresta Ombrófila Densa que pertencem à Serra do Mar: Estação Ecológica de Boracéia (94 espécies) (Yamamoto 1999); Núcleo Juréia (73 espécies) (Tavares 2002), Núcleo Cubatão (69 espécies) (Tavares 2002), Núcleo Cunha (79 espécies) (Tavares 2002); Parque Estadual Carlos Botelho (110 espécies) (Silva \& Brandão 2010) e Parque das Neblinas, que é uma Reserva Privada do Patrimônio Natural (87 espécies) (Suguituru et al. 2011). A comparação do número de espécies é diretamente possível, pois, em todos os remanescentes, as coletas foram efetuadas usando o mesmo protocolo.

Fatores como produtividade primária (Kaspari et al. 2000), complexidade e heterogeneidade do ambiente expressa pela riqueza e composição das comunidades de plantas (Ribas et al. 2003, Lassau \& Hochuli 2004), temperatura e umidade (King et al. 1998, Sabu et al. 2008) e topografia (Vasconcelos et al. 2003) podem ser responsáveis pela variação da riqueza observada nas diferentes localidades. Entretanto, em nenhuma área de Floresta Ombrófila Densa foi registrado um número tão baixo de espécie como é o caso da Fazenda Santo Alberto, localizada na Serra do Itapeti. Por outro lado, na Serra do Itapeti, também registramos a maior riqueza de espécies (Parque Natural Municipal Francisco Affonso de Mello), incluindo uma espécie ainda não descrita de predador especializado (Strumigenys sp.9). A alta diversidade $\alpha$ de formigas de serapilheira no Parque Natural Municipal Francisco Affonso de Mello está, possivelmente, relacionada à riqueza da vegetação na área do Parque e suas adjacências (Tomasulo 2012). A estrutura da vegetação é frequentemente um dos determinantes da estrutura de 


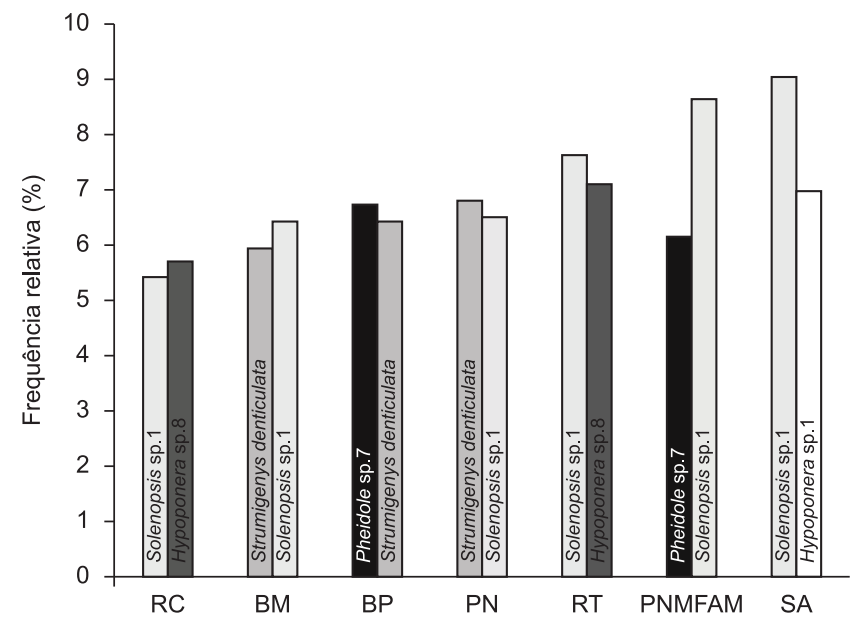

Figura 2. Espécies de formigas de serapilheira mais frequentes nos remanescentes de Floresta Ombrófila Densa da Bacia Hidrográfica do Alto Tietê.

Figure 2. Litter ant species most frequent in the remnants of Rain Forest of the Upper Tietê River Basin.

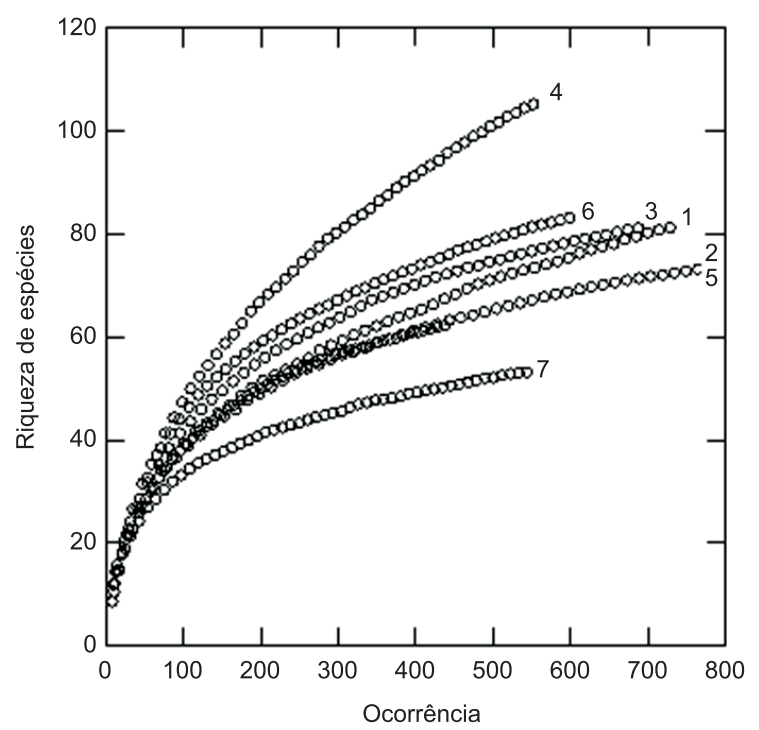

Figura 3. Comparação entre a riqueza de espécies entre os remanescentes florestais da Bacia Hidrográfica do Alto Tietê. (1) Barragem de Paraitinga; (2) Barragem de Biritiba Mirim; (3) Barragem de Ponte Nova; (4) Parque Natural Municipal Francisco Affonso de Mello; (5) Parque Estadual Nascentes do Rio Tietê; (6) Barragem de Ribeirão do Campo e (7) Fazenda Santo Alberto.

Figure 3. Comparison of species richness among forest remnants of Rain Forest of the Upper Tietê River Basin. (1) Paraitinga Dam; (2) Biritiba Mirim Dam; (3) Ponte Nova Dam; (4) Municipal Natural Park Francisco Affonso de Mello; (5) State Park Nascentes do Rio Tietê; (6) Ribeirão do Campo Dam and (7) Santo Alberto Farm.

comunidades de formigas (Ribas \& Schoereder 2002, Cardoso et al. 2010, Resende et al. 2011).

O Parque Estadual das Nascentes do Rio Tietê, apesar de representar um local de elevado valor ecológico, pois protege diversas nascentes que irão formar o mais importante rio do Estado de São Paulo, possui uma baixa diversidade e a menor densidade de espécies. Sugerimos que dois fatores são determinantes dos processos locais: (1) a recente implementação da área da nascente do Rio Tietê como Unidade de Conservação e (2) a maior parte do local é constituída

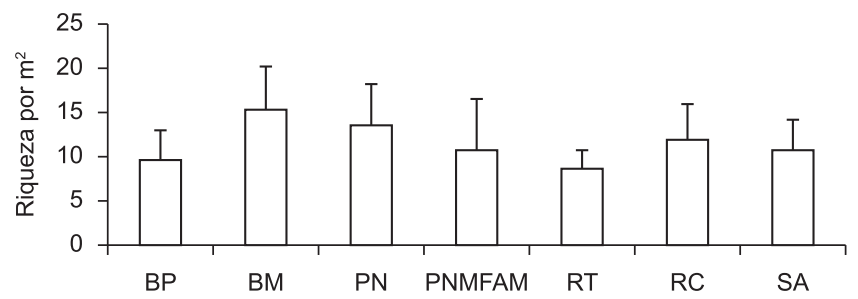

Figura 4. Número médio $\left( \pm\right.$ dp) de espécies de formigas por $\mathrm{m}^{2}$ de serapilheira de acordo com os remanescentes de Floresta Ombrófila Densa, da Bacia Hidrográfica do Alto Tietê. (BP) Barragem de Paraitinga; (BM) Barragem de Biritiba Mirim; (PN) Barragem de Ponte Nova; (PNMFAM) Municipal Natural Park Francisco Affonso de Mello; (RT) State Park Nascentes do Rio Tietê; (RC) Barragem de Ribeirão do Campo e (SA) Fazenda Santo Alberto. A barra vertical em cada coluna corresponde ao desvio padrão.

Figure 4. Mean number $\left( \pm \mathrm{sd}\right.$ ) of ant species per $\mathrm{m}^{2}$ of litter according to the remnants of Rain Forest of the Upper Tietê River Basin. (BP) Paraitinga Dam; (BM) Biritiba Mirim Dam; (PN) Ponte Nova Dam; (PNMFAM) Municipal Natural Park Francisco Affonso de Mello; (RT) State Park Nascentes do Rio Tietê; (RC) Ribeirão do Campo Dam and (SA) Santo Alberto Farm. Vertical bar at each column corresponds to standard deviation.

por Eucaliptus spp., com diferentes níveis de subbosque, o que leva a formação de uma serapilheira com elevados teores de tanino e um efeito negativo na composição de artrópodes de solo (Pozo et al. 1997).

Delabie et al. (2000) estudando sistemas agroflorestais de plantio de cacau, e com o mesmo protocolo de coleta de formigas de serapilheira, registraram de 5 a 12 espécies por $\mathrm{m}^{2}$, o que é similar aos resultados observados para o Parque Estadual Nascentes do Rio Tietê. Entretanto, o número de espécies por $\mathrm{m}^{2}$ registrado em todos os remanescentes do Alto Tietê foi menor quando comparado às áreas de Mata Atlântica estudadas por Silva \& Brandão (2010). Esses autores encontraram valores que variaram de $16,76 \pm 3,72$ a 20,78 $\pm 4,19$ espécies $/ \mathrm{m}^{2}$, sendo que em São Bento do Sul (Santa Catarina) foram registradas até 30 espécies $/ \mathrm{m}^{2}$. Contudo, independentemente da localidade, $S$. denticulata aparece como uma das espécies mais frequentes, o que corrobora Silva \& Brandão (2010).

Espécies com morfologia e biologia especializadas (Brandão et al. 2009), com diversidade reduzida em ambientes antropizados (Pacheco et al. 2009), também foram registradas, porém não em todas as áreas. Na área de mata de Biritiba Mirim encontra-se o maior número de espécies especialistas, com mandíbulas modificadas para predar dipluros campodeídeos (Prionopelta antillana), Myriapoda (Stigmatomma armigera e Stigmatomma elongatum) e ovos de artrópodes (Discothyrea sexarticulata) (Delabie et al. 2000, Brown Junior 2000). Na Fazenda Santo Alberto, apenas uma espécie, D. sexarticulata, foi registrada. Porém, outras técnicas de coleta devem ser empregadas nas áreas de floresta do Alto Tietê para registrar táxons especialistas da região. Por exemplo, a lista de espécie apresentada neste trabalho deve ser acrescentada Acanthostichus quadratus, que foi registrada no Parque Natural Municipal Francisco Affonso de Mello usando armadilhas subterrâneas (Morini et al. 2004), ou então, Labidus mars (Zara et al. 2003); ambas são espécies criptobióticas (Pacheco \& Vasconcelos 2012).

Outros estratos também devem ser analisados, como sementes (Silva et al. 2009) e troncos/galhos caídos na serapilheira (Carvalho \& Vasconcelos 2002, Fernandes et al. 2012). Determinadas espécies especialistas nidificam nesses locais, como é o caso de Typhlomyrmex rogenhoferi (Lacau et al. 2004, Delabie et al. 2007, Brandão et al. 2009) registrada na serapilheira das áreas de Floresta Ombrófila Densa da Barragem de Biritiba Mirim e do Parque Estadual Nascentes do 
Tabela 3. Resultados do índice de Whittaker obtidos com os dados do inventário de formigas na Bacia Hidrográfica do Alto Tietê.

Table 3. Results obtained from Whittaker index of inventory data of ants in the upper Tietê River Basin.

\begin{tabular}{ccccccc}
\hline & BP & BM & PN & PNMFAM & RT & RC \\
\hline BP & - & & & & & \\
BM & $0,35(51)$ & - & & & & \\
PN & $0,29(52)$ & $0,31(53)$ & - & & & \\
PNMFAM & $0,29(62)$ & $0,28(57)$ & $0,33(61)$ & - & & - \\
RT & $0,39(38)$ & $0,32(41)$ & $0,42(40)$ & $0,40(50)$ & - & $0,38(43)$ \\
RC & $0,32(47)$ & $0,32(47)$ & $0,41(48)$ & $0,35(60)$ & $0,35(47)$ & - \\
SA & $0,38(39)$ & $0,36(39)$ & $0,43(37)$ & $0,43(44)$ & $0,37(35)$ & 0 \\
\hline
\end{tabular}

Entre parênteses, o número de espécies compartilhadas entre os remanescentes.

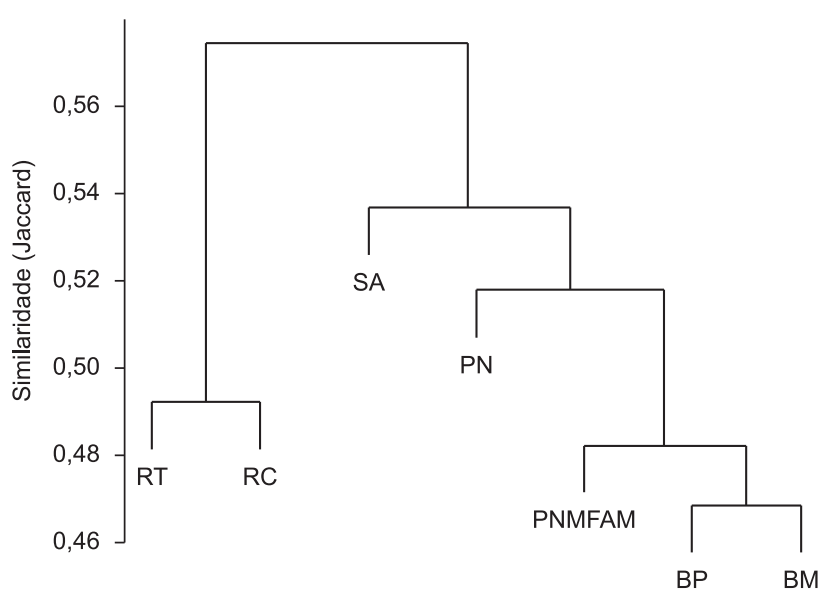

Figura 5. Similaridade entre as comunidades de formigas de serapilheira nos remanescentes de Floresta Ombrófila Densa da Bacia Hidrográfica do Alto Tietê. (BP) Barragem de Paraitinga; (BM) Barragem de Biritiba Mirim; (PN) Barragem de Ponte Nova; (PNMFAM) Parque Natural Municipal Francisco Affonso de Mello; (RT) Parque Estadual Nascentes do Rio Tietê; (RC) Barragem de Ribeirão do Campo e (SA) Fazenda Santo Alberto.

Figure 5. Similarity among litter ant communities in remnants of Rain Forest of the Upper Tietê River Basin. (BP) Paraitinga Dam; (BM) Biritiba Mirim Dam; (PN) Ponte Nova Dam; (PNMFAM) Municipal Natural Park Affonso de Mello; (RT) State Park Nascentes do Rio Tietê; (RC) Ribeirão do Campo Dam and (AS) Santo Alberto Farm.

Rio Tietê. Espécies de Myrmelachista, um gênero arborícola que faz parte da diversidade da serapilheira da Mata Atlântica da Serra do Mar (Suguituru et al. 2011), foram registradas em galhos caídos na serapilheira na Barragem de Ponte Nova, Parque Natural Municipal Francisco Affonso de Mello e Parque Estadual Nascentes do Rio Tietê (Nakano et al. 2012).

Na serapilheira dos remanescentes de Floresta Ombrófila Densa na Bacia Hidrográfica do Alto Tietê não foi registrada nenhuma espécie exótica. Todos os remanescentes de Mata Atlântica estão cercados por propriedades rurais ou pelas cidades de Salesópolis, Biritiba Mirim e Mogi das Cruzes, o que facilita o acesso de espécies que possuem o potencial de reduzir a fauna nativa, como é o caso de Paratrechina longicornis e Pheidole megacephala (Nafus 1993, Harris \& Barker 2007, Vanderwoude et al. 2000, Hoffmann 2010). Essas espécies exóticas são frequentes nas áreas urbanas das cidades do Alto Tietê (Kamura et al. 2007, Munhae et al. 2009, Souza et al. 2012). Por outro lado, ocorre uma forte dominância de Solenopsis sp. 1 em $86 \%$ dos remanescentes. Este gênero é composto por espécies muito agressivas, inclusive na serapilheira (Delabie \& Fowler 1995). Essa dominância pode estar relacionada às mudanças na estrutura da vegetação natural (Resende et al. 2011), o que possivelmente se deve às fortes pressões ambientais que o Alto Tietê está sofrendo; em especial do setor imobiliário e moradias clandestinas (Bruna et al. 2012). Vários estudos demonstram que a fragmentação causa uma redução na riqueza de espécies e mudanças profundas na composição de espécies (Vasconcelos 2003).

Nossos resultados mostram que a composição de espécies entre os remanescentes é semelhante, o que possivelmente está relacionado à similaridade florística e a distância entre as áreas (Wolda 1996). Os valores de espécies compartilhadas mostram a importância dos remanescentes estudados para a conservação da diversidade de formigas da Bacia Hidrográfica do Alto Tietê. Neste caso, destacase o remanescente de Floresta Ombrófila Densa onde se localiza o Parque Natural Municipal Francisco Affonso de Mello, que detém os maiores valores de diversidade $\alpha$ e $\beta$ e, por consequência, alta contribuição para a diversidade $\gamma$ (Chase et al. 2011).

Os nossos resultados expandem o atual conhecimento sobre as espécies de formigas de serapilheira da Bacia Hidrográfica do Alto Tietê e medidas que visem à conservação, proteção e conectividade dos recursos naturais (Brasil 2000) devem ser efetuadas, principalmente no Parque Estadual das Nascentes do Rio Tietê, que ainda possui muitos eucaliptos e apresenta baixos valores de diversidade $\alpha$ e $\beta$. Os resultados também mostram (1) o Parque Natural Municipal Francisco Affonso de Mello como o mais rico remanescente de Floresta Ombrófila Densa da região, corroborando diversos trabalhos apresentados por Morini \& Miranda (2012); (2) a importância das áreas de Floresta Ombrófila Densa localizadas nas adjacências das barragens para a manutenção da biodiversidade de formigas; biodiversidade essa ameaçada pelo cenário socioambiental vigente na região (Candido et al. 2010) e (3) a substituição de espécies entre os remanescentes, o que indica a importância dessas áreas para a conservação da fauna regional de formigas da Bacia Hidrográfica do Alto Tietê.

\section{Agradecimentos}

À FAPESP (Processos n. 98/14509-0 e 05/58556-8), à FAEP/ UMC, ao Cnpq (Processo n. 301151/2009-1) pelo auxílio financeiro e ao Dr. Rogério Rosa Silva pela identificação das espécies.

\section{Referências}

AGOSTI, D. \& ALONSO, L.E. 2000. The ALL Protocol: a standard protocol for the collection of ground-dwelling ants. In Ants: standard methods for measuring and monitoring biodiversity (D. Agosti, J.D. Majer, L.E. Alonso \& T.R. Schultz, eds.). Smithsonian Institution Press, Washington, p.204-206.

AGOSTI, D., MAJER, J.D., ALONSO, L.E. \& SCHULTZ, T.R. 2000. The biodiversity Challenge. In Ants: standard methods for measuring and monitoring biodiversity (D. Agosti, J.D. Majer, L.E. Alonso \& T.R. Schultz, eds.). Smithsonian Institution Press, Washington, p.17-19. 
ALONSO, L.E. \& AGOSTI, D. 2000. Biodiversity studies, monitoring, and ants: an overview. In Ants: standard methods for measuring and monitoring biodiversity (D. Agosti, J.D. Majer, L.E. Alonso \& T.R. Schultz, eds.). Smithsonian Institution Press, Washington, p.1-8.

ANDERSEN, A.N. 1983. Species diversity and temporal distribution of ants in the semi-arid mallee region of northwestern Victoria. Austral J. Ecol. 8:127-137. http://dx.doi.org/10.1111/j.1442-9993.1983.tb01600.x

ANDERSEN, A.N. \& MAJER, J.N. 2004. Ants show the way down under: invertebrates as bioindicators in land management. Front. Ecol. Environ. 2:291-298. http://dx.doi.org/10.1890/1540-9295 (2004)002[0292:ASTWDU]2.0.CO;2

ARMBRECHT, I., PERFECTO, I. \& VANDERMEER, J. 2004. Enigmatic biodiversity correlations: ant diversity responds to diverse resources. Science 304:284-286. http://dx.doi.org/10.1126/science.1094981

AYRES, M., AYRES, M.J., AYRES, D.L. \& SANTOS, A.S. 2007. BioEstat 5.0: aplicações estatísticas nas áreas das ciências biológicas e médicas. Instituto de Desenvolvimento Sustentável Mamirauá - IDSM/ $\mathrm{MCT} / \mathrm{CNPq}, 364 \mathrm{p}$

BESTELMEYER, B.T., AGOSTI, D., ALONSO, L.E., BRANDÃO, C.R.F., BROWN JR, W.L., DELABIE, J.H.C. \& SILVESTRE, R. 2000. Field techniques for the study of ground-dwelling ants. In Ants: standard methods for measuring and monitoring biodiversity (D. Agosti, J.D. Majer, L.E. Alonso \& T.R. Schultz, eds.). Smithsonian Institution Press, Washington, p.22-144.

BOLTON, B. 2003. Synopsis and classification of Formicidae. Memoirs AEI 71:1-370.

BOLTON, B., ALPERT, G., WARD, F.S. \& NASKRECKI, P. 2006. Bolton's catalogue of ants of the world: 1758-2005. Harvard University Press, Cambridge, Massachusetts. http://gap.entclub.org/.

BRANDÃO, C.R.F., SILVA, R.R. \& DELABIE, J.H.C. 2009. Formigas (Hymenoptera). In Bioecologia e Nutrição de Insetos: base para o manejo integrado de pragas (A.R. Panizzi \& J.R.P. Parra). Embrapa Tecnológica, Brasília, 1164p.

BRASIL. Ministério do Meio Ambiente. 2000. Lei n. 9.985, de 18 de julho de 2000. Regulamenta o art. 225, §1o, incisos I, II, III e VII da Constituição Federal, institui o Sistema Nacional de Unidades de Conservação da Natureza e dá outras providências. Diário Oficial da República Federativa do Brasil, Brasília, 19 jul. 2000.

BROWN JUNIOR, W.L. 2000. Diversity of ants. In Ants: standard methods for measuring and monitoring biodiversity (D. Agosti, J.D. Majer, L.E. Alonso \& T.R. Schultz, eds.). Smithsonian Institution Press, Washington, p.45-79.

BRUnA, G.C., ALMEIDA, M.A.P., SAntos, M.V.M \& YAMAMOTO,S.L. 2012. Degradação da Serra do Itapeti. In Serra do Itapeti: aspectos históricos, sociais e naturalísticos (M.S.C.Morini \& V.F.O.Miranda, org.). Editora Canal6, Bauru, p.59-73.

CÂMARA, I.G. 2003. Brief history of conservation in the Atlantic forest. In The Atlantic Forest of South America: biodiversity status, threats, and outlook (C. Galindo-Leal \& I.G. Câmara, eds.). Center for Applied Biodiversity Science e Island Press, Washington, p. 31-42.

CAMPOS, R.F., SCHOEREDER, J.H. \& SPERBER, C.F. 2003. Local determinants of species richness in litter ant communities (Hymenoptera: Formicidae). Sociobiology 41:357-367.

CANDIDO, M.S., FONSECA, A.C.R., \& DANTAS, V.M. 2010. Protocolo em defesa da recuperação da qualidade socioambiental da bacia hidrográfica do alto Tietê Cabeceiras, $94 \mathrm{p}$.

CARDOSO, D.C., SOBRINHO, T.G. \& SCHOEREDER, J.H. 2010. Ant community composition and its relationship with phytophysiognomies in a Brazilian Restinga. Insects Soc. 57:293-301. http://dx.doi.org/10.1007/ s00040-010-0084-3

CARNAVAL, A.C., HICKERSON, M.J., HADDAD, C.F.B., RODRIGUES, M.T. \& MORITZ, C. 2009. Stability predicts genetic diversity in the Brazilian Atlantic Forest Hotspot. Science 323:785-789. http://dx.doi. org $/ 10.1126 /$ science. 1166955
CARVALHO, K.S. \& VASCONCELOS, H.L. 1999. Forest fragmentation in central Amazonian and its effects on litter-dwelling ants. Biol. Conserv. 20:151-157. http://dx.doi.org/10.1016/S0006-3207 (99)00079-8

CARVALHO, K.S. \& VASCONCELOS, H.L. 2002. Comunidade de formigas que nidificam em pequenos galhos da serapilheira em floresta da Amazônia Central, Brasil. Ver. Bras. Entomol. 46:115-121. http://dx.doi. org/10.1590/S0085-56262002000200002

CETESB. 1995. Relatório de qualidade das águas interiores do estado de São Paulo. Série de Relatório, n.2-3.

CETESB. 1999. Estabelecimento de valores de referência de qualidade e de intervenção para solos e águas subterrâneas no estado de São Paulo. Documentos Ambientais, n.1 e 2.

CHASE, J.M., KRAFT, N.J.B., SMITH, K.G., VELLEND, M. \& INOUYE, B. 2011. Using null models to disentangle variation in community from variation in $\alpha$-diversity. Ecosphere 22:1-11.

COLWELL, R.K. 2009. EstimateS: statistical estimation of species richness and shared species from samples. version 8.2. (último acesso em 08/07/2012).

DELABIE, J.H. \& FOWLER, H.G. 1995. Soil and litter cryptic ant assemblages of Bahian cocoa plantations. Pedobiologia 39:423-433.

DELABIE, J.H., AGOSTI, D. \& NASCIMENTO, I.C. 2000. Litter ant communities of the Brazilian Atlantic rain Forest region. In Sampling ground-dwelling ants: case studies from the word's rain forests (D. Agosti, J.D. Majer, L.E. Alonso \& T.R. Schultz, eds.). Curtin University, School of Environmental Biology Bulletin 18, Perth, p.1-17.

DELABIE, J.H.C., JAHYNY, B., NASCIMENTO, I.C., MARIANO, S.F., LACAU, S., CAMPIOLO, S., PHILPOTT, S.M. \& LEPONCE, M. 2007. Contribution of cocoa plantations to the conservation of native ants (Insecta: Hymenoptera: Formicidae) with a special emphasis on the Atlantic Forest fauna of southern Bahia, Brazil. Biodivers. Conserv. 16:2359-2384. http://dx.doi.org/10.1007/s10531-007-9190-6

DEL-CLARO, K. \& OLIVEIRA, P.S. 1999. Ant-homoptera interactions in a Neotropical Savanna: the honeydew-producing treehopper, Guayaquila xiphias (Membracidae), and its associated ant fauna on Didymopanax vinosum (Araliaceae). Biotropica 31:135-144.

FERNANDES, T.T., SILVA, R.R., SOUZA, D.R., ARAUJO, N. \& MORINI, M.S.C. 2012. Undecomposed twigs in the leaf litter as nest-building resources for ants (Hymenoptera: Formicidae) in areas of the Atlantic Forest in the southeastern region of Brazil. Psyche. (no prelo).

FOLGARAIT, P.J. 1998. Ant biodiversity and its relationship to ecosystem functioning: a review. Biodivers. Conserv. 7:1221-1244. http://dx.doi. org/10.1023/A:1008891901953

FUNDAÇÃO SOS MATAATLÂNTICA. 1998. Evolução dos remanescentes florestais e ecossistemas associados do domínio Mata Atlântica no período 1990-1995. INPE, São Paulo.

GOTELLI, N.J. 2009. Ecologia. Editora Planta, Londrina, Paraná, 287p.

HOLE, F.D. 1981. Effects of animals on soil. Geoderma 25:75-112. http:// dx.doi.org/10.1016/0016-7061 (81)90008-2

HARRIS, R.J. \& BARKER, G. 2007. Relative risk of invasive ants (Hymenoptera: Formicidae) establishing in New Zealand. New Zealand J. Zool. 34:161-178. http://dx.doi.org/10.1080/03014220709510075

HOFFMANN, B.D. 2010. Ecological restoration following the local eradication of an invasive ant in Northern Australia. Biol. Invasions 12:959-969. http://dx.doi.org/10.1007/s10530-009-9516-2

HÖLLDOBLER, B. \& WILSON, E.O. 1990. The ants. Belknap Press, Cambridge, Massachussets, 732p.

JOLY, C.A., AIDAR, M.P.M., KLINK, C.A., McGRATH, D.G., MOREIRA, A.G., MOUTINHO, P., NEPSTAD, D.C., OLIVEIRA, A.A., POTT, A., RODAL, M.J.N. \& SAMPAIO, E.V.S.B. 1999. Evolution of the Brazilian phytogeography classification systems: implications for biodiversity conservation. Ciênc. Cult. 51:331-348.

JOLY, C.A., LEITÃO-FILHO, H.F. \& SILVA, S.M. 1992. O patrimônio florístico. In Mata Atlântica (I.G. Câmara, coord.). Index, Rio de Janeiro, p.96-128. 
KAMURA, C.M., MORINI, M.S.C., FIGUEIREDO, C.J., BUENO, O.C. \& CAMPOS-FARINHA, A.E.C. 2007. Ant communities (Hymenoptera: Formicidae) in an urban ecosystem near the Atlantic Rainforest. Braz. J. Biol. 67:635-641. http://dx.doi.org/10.1590/S1519-69842007000400007

KASPARI, M. 1996. Testing resource-based models of patchiness in four Neotropical litter ant assemblages. Oikos 76:443-454. http://dx.doi. org $/ 10.2307 / 3546338$

KASPARI, M. \& WEISER, M.D. 2000. Ant activity along moisture gradients in a Neotropical forest. Biotropica 32:703-711. http://dx.doi. org/10.1646/0006-3606 (2000)032[0703:AAAMGI]2.0.CO;2

KASPARI, M., O'DONNELL, S. \& KERCHER, J.R. 2000. Energy, density, and constraints to species richness: ant assemblages along a productivity gradient. Am. Nat. 155:280-293. http://dx.doi.org/10.1086/303313

KING, J.R., ANDERSEN, A.N. \& CUTTER, A.D. 1998. Ant as bioindicators of habitat disturbance: validation of the functional group model for Australia`s humid tropics. Biodiv. Conserv. 7:1627-1638. http://dx.doi. org/10.1023/A:1008857214743

LACAU, S., VILLEMANT, C. \& DELABIE, J.H.C. 2004. Typhlomyrmex meire, a remarkable new species endemic to Southern Bahia, Brazil (Formicidae: Ectatomminae). Zootaxa 678:1-23.

LAPOLLA, J., BRADY, S. \& SHATTUCK, S. 2010. Phylogeny and taxonomy of the Prenolepis genus group of ants (Hymenoptera: Formicidae). Syst. Entomol. 35:118-131. http://dx.doi.org/10.1111/ j.1365-3113.2009.00492.x

LASSAU, S.A. \& HOCHULI, D.F. 2004. Effects of habitat complexity on ant assemblage. Ecography 27:157-164. http://dx.doi.org/10.1111/j.09067590.2004.03675.x

LAVELLE, P., BIGNELL, D., LEPAGE, M.,WOLTERS,V., ROGER, P., INESON, P., HEAL, O.W. \& DHILLION, S. 1997. Soil functions in a changing world: the role of invertebrate ecosystem engineers. European J. Soil Biol. 33:159-193.

MAJER, J.D. 1983. Ants: bioindicators of minesite rehabilitation, land-use, and land conservation. Environ. Manage. 7:375-383.

MAJER, J.D., BRENNAN K.E.C. \& MOIR, M.L. 2007. Invertebrates and the restoration of a forest ecosystem: 30 years of research following bauxite mining in Western Australia. Restor. Ecol. 15:104-115. http:// dx.doi.org/10.1007/BF01866920

MARCENIUK, A.P. \& HILSDORF, A.W.S. 2010. Peixes: das cabeceiras do rio Tietê e Parque das Neblinas. Editora Canal6, Bauru, SP, 160p.

MARTINI, A.M.Z., FIASCHI, P., AMORIM, A.M. \& PAIXÃO, J.L. 2007. A hot-point within a hotspot: a high diversity site in Brazil's Atlantic Forest. Biodivers. Conserv. 16:3111-3128. http://dx.doi.org/10.1007/ s10531-007-9166-6

McKEY, D., ROSTAIN, S., IRIARTE, J., GLASER, B., BIRK, J.J., HOLST, I. \& RENARD, D. 2010. Pre-columbian agricultural landscapes, ecosystem engineers, and self-organized patchiness in Amazonia. PNAS 107:78237828. http://dx.doi.org/10.1073/pnas.0908925107

METZGER, J.P., MARTENSEN, A.C., DIXO, M., BERNACCI, L.C., RIBEIRO, M.C., TEIXEIRA, A.M.G. \& PARDINI, R. 2009. Time-lag in biological responses to landscape changes in a highly dynamic Atlantic forest region. Biol. Conserv. 142:1166-1177. http://dx.doi.org/10.1016/j. biocon.2009.01.033

MINUZZI, R.B., SEDIYAMA, G.C., BARBOSA, E.M. \& MELO JUNIOR, J.C.F. 2007. Climatologia do comportamento do período chuvoso da região sudeste do Brasil. Rev. Bras. Meteorol. 22:338-344.

MORINI, M.S.C. \& MIRANDA, V.F.O. 2012. Serra do Itapeti: aspectos históricos, sociais e naturalísticos. Editora Canal6, Bauru, p.398.

MORINI, M.S.C., YASHIMA, M., ZENE, F.Y., SILVA, R.R. \& JAHYNY, B. 2004. Observations on the Acanthostichus quadratus (Hymenoptera: Formicidae: Cerapachyinae) visiting underground bait and fruits of the Syagrus romanzoffiana, in an area of the Atlantic Forest, Brazil. Sociobiology 43:573-578.

MUNHAE, C.B., BUENO, Z.A.F.N., MORINI, M.S.C. \& SILVA, R.R. 2009 Composition of the ant fauna (Hymenoptera: Formicidae) in public squares in Southern Brazil. Sociobiology 53:455-472.
MYERS, N., MITTERMEIER, R.A., MITTERMEIER, C.G., FONSECA, G.A.B. \& KENT, J. 2000. Biodiversity hotspots for conservation priorities. Nature 403:853-858. http://dx.doi.org/10.1038/35002501

NAFUS, D.M. 1993: Movement of introduced biological control agents onto nontarget butterflies, Hypolimnas spp. (Lepidoptera: Nymphalidae). Environ. Entomol. 22:265-272.

NAKANO, M.A., FEITOSA, R.M., MORAES, C.O., ADRIANO, L.D.C., HENGLES, E.P., LONGUI, L.E. \& MORINI, M.S.C. 2012. Assembly of Myrmelachista Roger (Formicidae: Formicinae) in twigs fallen on the leaf litter of Brazilian Atlantic Forest. J. Nat. Hist. http://dx.doi.org/10. 1080/00222933.2012.707247

NOGUEIRA, I.S., NABOUT, J.C., OLIVEIRA, J.E. \& SILVA, K.D. 2008 Diversidade (alfa, beta e gama) da comunidade fitoplanctônica de quatro lagos artificiais urbanos do município de Goiânia, GO. Hoehnea 35:219233.

OKSANEN, J., BLANCHET, F.G., KINDT, R., LEGENDRE, P., O'HARA, R.B., SIMPSON, G.L., STEVENS, M.H.H. \& WAGNER, H. 2011. Vegan: community ecology package. Version $1.17-11$. http://vegan.rforce.r-project.org/.

PACHECO, R. \& VASCONCELOS, H.L. 2012. Subterranean pitfall traps: is it worth including them in your ant sampling protocol? Psyche. http:// dx.doi.org/10.1155/2012/870794

PACHECO, R., SILVA, R.R., MORINI, M.S.C. \& BRANDÃO, C.R.F. 2009. A comparison of the leaf-litter ant fauna in a secondary Atlantic forest with an adjacent pine plantation in southeastern Brazil. Neotrop. Entomol. 38:5565. http://dx.doi.org/10.1590/S1519-566X2009000100005

PAGANI, M.I. 2012. Preservação da Serra do Itapeti. In Serra do Itapeti: aspectos históricos, sociais e naturalísticos (M.S.C.Morini \& V.F.O.Miranda, org.). Editora Canal6, Bauru, p.45-58.

PAIS, M.P. \& VARANDA, E.M. 2010. Arthropod recolonization in the restoration of a semideciduous forest in southeastern Brazil. Neotrop. Entomol. 39:198-206. http://dx.doi.org/10.1590/S1519. 566X2010000200009

PHILPOTT, S.M. \& FOSTER, P.F. 2005. Nest-site limitation in coffee agroecossystems: artificial nest promote maintenance of arboreal ant diversity. Ecol. Appl. 15:1478-1485. http://dx.doi.org/10.1890/04-1496

POZO, J., GONZÁLES, E., DÍEZ, J.R., MOLINERO, J. \& ELÓSEGUI, A. 1997. Inputs of particulate organic matter to streams with different riparian vegetation. J. North Am. Benthol. Soc. 16:602-611. http://dx.doi. org/10.2307/1468147

RESENDE, J.J., SANTOS, G.M.M., NASCIMENTO, I.N., DELABIE, J.H.C. \& SILVA, E.M.S. 2011. Communities of ants (Hymenoptera: Formicidae) in different Atlantic Rain Forest phytophysionomies. Sociobiology 58:779-798.

RIBAS, C.R. \& SCHOEREDER, J.H. 2002. Are all mosaics caused by competition? Oecologia 131:606-611. http://dx.doi.org/10.1007/s00442002-0912-x

RIBAS, C.R., SCHOEREDER, J.H., PIE, M. \& SOARES, S.M. 2003. Tree heterogeneity, resource availability, and larger scale processes regulating arboreal ant species richness. Austral Ecol. 28:305-314. http://dx.doi. org/10.1046/j.1442-9993.2003.01290.x

RIBEIRO, M.C., METZGER, J.P., MARTENSEN, A.C., PONZONI, F. \& HIROTA, M.M. 2009. Brazilian Atlantic forest: how much is left and how is the remaining forest distributed? Implications for conservation. Biol. Conserv. 142: 1141-1153. http://dx.doi.org/10.1016/j.biocon.2009.02.021

RISCH, A.C. \& JURGENSEN, M.F. 2008. Ants in the soil system - a hydrological, chemical and biological approach. J. Appl. Entomol. 132:265. http://dx.doi.org/10.1111/j.1439-0418.2008.01296.x

SABU, T.K., VINEESH, P.J. \& VINOD, K.V. 2008. Diversity of forest litterinhabiting ants along elevations in the Wayanad region of the Western Ghats. J. Ins. Sci. 8:1-14. http://dx.doi.org/10.1673/031.008.6901

SANDERS, D. \& VAN VEEN, F.F. 2011. Ecosystem engineering and predation: the multi-trophic impact of two ant species. J. Animal Ecol. 80:569-576. http://dx.doi.org/10.1111/j.1365-2656.2010.01796.x 
SILVA, R.R. \& BRANDÃO, C.R.F. 2010. Morphological patterns and community organization in leaf-litter assemblages. Ecol. Monog. 80:107124. http://dx.doi.org/10.1890/08-1298.1

SILVA, F.R., BEGNINI, R.M., KLIER, V.A., SCHERER, K.Z., LOPES, B.C. \& CASTELLANI, T.T. 2009. Utilização de sementes de Syagrus romanzoffiana (Arecaceae) por formigas em floresta secundária no sul do Brasil. Neotrop. Entomol. 38:873-875. http://dx.doi.org/10.1590/ S1519-566X2009000600025

SOUZA, D.R., MUNHAE,C.B., KAMURA,C.M., PORTERO,N.S. \& MORINI, M.S.C. 2012. Formigas em áreas urbanizadas da Serra do Itapeti. In Serra do Itapeti: aspectos históricos, sociais e naturalísticos (M.S.C.Morini \& V.F.O.Miranda, org.). Editora Canal6, Bauru, p.301310.

STEPHENS, S.S. \& WAGNER, M.R. 2006. Using ground foraging ant (Hymenoptera: Formicidae) functional groups as bioindicators of forest health in Northern Arizona ponderosa pine forests. Environ. Entomol. 35:937-949. http://dx.doi.org/10.1603/0046-225X-35.4.937

SUGUITURU, S.S., SILVA, R.R., SOUZA, D.R., MUNHAE, C.B. \& MORINI, M.S.C. 2011. Ant community richness and composition across a gradient from Eucalyptus plantations to secondary Atlantic Forest. Biota Neotrop. 11:1-8. http://dx.doi.org/10.1590/S1676-06032011000100034

TAVARES, A.A. 2002. Estimativas da diversidade de formigas (Hymenoptera: Formicidae) de serapilheira em quatro remanescentes de floresta ombrófila densa e uma restinga no estado de São Paulo, Brasil. Tese de doutorado, Faculdade de Filosofia, Ciências e Letras de Ribeirão Preto da USP, Entomologia, São Paulo.

TOMASULO, P.L.B. 2012. Flora fanerogâmica da Serra do Itapeti. In Serra do Itapeti: aspectos históricos, sociais e naturalísticos (M.S.C.Morini \& V.F.O.Miranda, org.). Editora Canal6, Bauru, p.107-125.
VANDERWOUDE, C., LOBRY DE BRUYN, L.A. \& HOUSE, A.P.N. 2000. Response of an open-forest ant community to invasion by the introduced ant, Pheidole megacephala. Austral Ecol. 25:253-259. http://dx.doi. org/10.1046/j.1442-9993.2000.01021.x

VASCONCELOS, H.L. 2003. Formigas do solo nas florestas da Amazônia: padrões de diversidade e respostas aos distúrbios naturais e antrópicos (F.M.S. Moreira, J.O. Siqueira \& L. Brussard, eds.). Editora da Universidade Federal de Lavras, Minas Gerais, p.323-343.

VASCONCELOS, H.L., MACEDO, A.C.C. \& VILHENA, J.M.S. 2003. Influence of topography on the distribution of ground-dwelling in a Amazonian forest. Stud. Neotrop. Fauna Environ. 38:115-124. http:// dx.doi.org/10.1076/snfe.38.2.115.15923

WARD, P.S. 2000. Broad-scale patterns of diversity in a leaf litter ant communities. In Ants: standard methods for measuring and monitoring biodiversity (D. Agosti, J.D. Majer, L.E. Alonso \& T.R. Schultz, eds.). Smithsonian Institution Press, Washington, p.99-121.

WHITTAKER, R.H. 1960. Vegetation of the Siskioy Mountains, Oregon and California. Ecol. Monog. 30:279-338. http://dx.doi.org/10.2307/1943563

WOLDA, H. 1996. Between-site similarity in species composition of a number of Panamanian insect group. Miscell. Zool. 19:39-50.

XAVIER, A.F., BOLZARI, B.M. \& JORDÃO, S. 2008. Unidade de conservação da natureza no Estado de São Paulo, unidade 3. In Diretrizes para a conservação e restauração da biodiversidade no Estado de São Paulo (R.R. Rodrigues \& V.L. Bononi, org). Instituto de Botânica, p.24.

YAMAMOTO, C.I. 1999. Fauna de formigas de serapilheira de Mata Atlântica: levantamento quantitativo na estação biológica de Boracéia (Salesópolis, SP). Disssertação de Mestrado, Instituto de Biociências, Universidade de São Paulo, 95p.

ZARA, F.J., MORINI, M.S.C. \& KATO, L.M. 2003. New record for the army ants Labidus mars (Formicidae: Ecitoninae) in atlantic rain forest in São Paulo State, Brazil. Sociobiology 42:443-448.

Recebido em 20/04/2011

Versão reformulada recebida em 07/02/2013

Publicado em 15/04/2013 